PoS $\quad \begin{aligned} & \text { PROCEEDINGS } \\ & \text { OF SCIENCE }\end{aligned}$

\title{
Nucleon Decay searches and Indirect Detection of Dark Matter with JUNO
}

\section{Wei Wang*}

Sun Yat-Sen University

E-mail: wang223@mail.sysu.edu.cn

\section{Suprabh Prakash}

Sun Yat-Sen University

E-mail: prakash3amail.sysu.edu.cn

38th International Conference on High Energy Physics 3-10 August 2016

Chicago, USA

$$
{ }^{*} \text { Speaker. }
$$


The Jiangmen Underground Neutrino Observatory (JUNO) is designed to resolve the neutrino mass hierarchy by observing the survival spectrum of antineutrinos from multiple nuclear reactors that are $\sim 53 \mathrm{~km}$ away in its $20 \mathrm{kt}$ liquid scintillator detector. To be able to resolve the subtle oscillation cycle shifts in the survival spectrum caused by different neutrino mass hierarchies, the JUNO detector has been designed to reach energy resolution of $3 \% / \sqrt{E / M e V}$ and energy scale uncertainty better than $1 \%$ for the energy range that is relevant for reactor antineutrinos. With such energy response performance, massive size, and $\sim 700 \mathrm{~m}$ rock overburden, the JUNO detector is potentially a competitive device that can be used to probe other outstanding problems beyond neutrino oscillation physics as well. In this work, we explore JUNO's potential to solve two such problems - 1) Nucleon Decay searches, and 2) Indirect Detection of Dark Matter.

Proton decays, via the kaon plus anti-neutrino channel are favored by many SUSY GUT models. The current lifetime has been constrained to be greater than $5.9 \times 10^{33}$ years at $90 \%$ C.L. by the massive Super-Kamiokande (Super-K) water Cerenkov detector. The JUNO detector will have a much higher detection efficiency, $\sim 65 \%$ preliminarily, for this particular channel due to its superior energy response compared to Super-K. Our preliminary analysis shows that it can surpass the current Super-K limit in 3 years of data taking. With 10 years of data, the expected background is $\sim 0.5$ events. If no event is observed, it can reach $1.9 \times 10^{34}$ years at $90 \%$ C.L., which is very close to the region of interest predicted by some GUT models. In the case of indirect detection of Dark Matter also, JUNO's performance is quite remarkable. We have considered the neutrino flux resulting from DM annihilation inside the Sun via the channels $\chi \chi \rightarrow \tau^{+} \tau^{-}$and $\chi \chi \rightarrow v \bar{v}$. Only the muon events with track lengths greater than $5 \mathrm{~m}$ within the detector have been considered. For such events, the direction of muon tracks can be reconstructed with an accuracy better than $1^{\circ}$. We find that JUNO's sensitivity to spin-dependent scattering cross-section $\sigma_{\chi p}^{S D}$ is much better than the current direct detection constraints. In the case of spin-independent $\sigma_{\chi p}^{S I}$ also, JUNO is competitive with direct detection experiments for $m_{\chi}<7 \mathrm{GeV}$. 